\title{
Power Control for Cognitive Radio Networks: A Game Theoretic Approach
}

\author{
Waqas Gulzar ${ }^{1}$ D . Abdullah Waqas ${ }^{2}$ - Hammad Dilpazir ${ }^{1,3}$ - Anwar Khan ${ }^{1}$. \\ Ashfaq Alam ${ }^{1} \cdot$ Hasan Mahmood ${ }^{1}$
}

Accepted: 16 September 2021 / Published online: 21 October 2021

(c) The Author(s) 2021

\begin{abstract}
In communication industry one of the most rapidly growing area is wireless technology and its applications. The efficient access to radio spectrum is a requirement to make this communication feasible for the users that are running multimedia applications and establishing real-time connections on an already overcrowded spectrum. In recent times cognitive radios (CR) are becoming the prime candidates for improved utilization of available spectrum. The unlicensed secondary users share the spectrum with primary licensed user in such manners that the interference at the primary user does not increase from a predefined threshold. In this paper, we propose an algorithm to address the power control problem for CR networks. The proposed solution models the wireless system with a noncooperative game, in which each player maximize its utility in a competitive environment. The simulation results shows that the proposed algorithm improves the performance of the network in terms of high SINR and low power consumption.
\end{abstract}

Keywords Cognitive radio networks · Wireless networks · Game theory · Power control

Waqas Gulzar

waqas.gulzar@phd.unict.it

Abdullah Waqas

abdullah@nutech.edu.pk

Hammad Dilpazir

hammad.dilpazir@numl.edu.pk

Anwar Khan

anwarkhanqau@gmail.com

Ashfaq Alam

ashfaq.vicky999@gmail.com

Hasan Mahmood

hasan@qau.edu.pk

1 Department of Electronics, Quaid-i-Azam University, Islamabad, Pakistan

2 Department of Electrical Engineering, National University of Technology, Islamabad, Pakistan

3 Present Address: Department of Electrical Engineering, National University of Modern Languages, Islamabad, Pakistan 


\section{Introduction}

The radio spectrum these days has been allotted on a dedicated basis. In other words, each wireless network gets an unique license to operate in a specific frequency band. The policy allows multiple users to transmit their signal over the dedicated channels at required transmission power without creating interference to other licensed users. [1]. The percentage utilization of allocated spectrum has been explored in various studies [2,3] and concluded that the assigned spectrum bands are underutilized in most of the cases. In order to increase the usage of available spectrum, cognitive radio networks (CRNs) allow the primary users (PUs) also known as licensed users and secondary users (SUs) also referred to as unlicensed users (or cognitive users) to share the available radio spectrum among themselves [4]. This spectrum sharing can be very fruitful in number of ways. For example, more than one wireless networks can access the same frequency band. Furthermore, this spectrum sharing technique allows operators to access spectrum more easily and can deploy their wireless infrastructure where it is required [5]. Therefore, cognitive radio networks helps in full utilization of available spectrum by efficient management of available frequency bands.

Cognitive radio is defined as smart and intelligent, re-configurable wireless communication system that is fully informed about its surrounding environment [6]. The main objective of this technology is the maximum utilization of the available spectrum of PUs while maintaining the quality of communication between the cognitive users (CU) under the condition that the interference to the PU does not increases from a threshold limit. In this dynamic way of spectrum sharing, we can check the capacitive gains when the channel varies due to fading effect, and the results are quite convincing. By adopting this technique, we can enhance the utilization of available spectrum [7]. To maintain the QoS requirement, it is necessary that the assigned channel is not over populated. Power control/channel assignment is carried out in such manners that the primary users are not affected by excessive interference. For CR networks power control is thoroughly studied in [8].

In [9], the author discussed some important rules to maximize the utilization of spectrum by selecting the optimal interference power and interference temperature constraint in CR networks. To obtain the ergodic capacity in fading channel for cognitive users, the strategies for optimal power allocation are studied in [10]. The results show that transmit and interference power can be restricted by peak/average constraint based on different combinations of power constraints. The gain in capacity for cognitive users also studied under the average peak transmit power or interference power constraint. In [11], a link gain pricing scheme was proposed with an error estimator $(\mathrm{H})$ for power control. The link gain pricing scheme helps to manage the fairness between the cognitive radio network. The applied filter estimates the channel variation [11]. Orthogonal frequency division multiplexing (OFDM) provides a very sufficient modulation technique for spectrum sharing. The use of OFDM-based cognitive radio is also very effective in maximizing the throughput of cognitive users and also minimizing the transmission power [12].

Game theory is a very useful mathematical tool in dealing with decentralized networks, where individual decision making is very critical to make the communication possible. It is also very useful in settling the conflicts between decision-makers in such type of selforganized systems.

For the existence of PUs in a frequency band along with the secondary users, the spectrumsharing is indispensable. In [13], the optimal rate and power are obtained by iteratively magnifying each CUs utility function, which is arranged to guarantee the conservation of primary user (PU) along with the QoS requirements of cognitive users. To access the open spectrum 
in a self organized network is a challenge, because of the conflicting interests of both primary and cognitive users. In [14], game theoretical approach was proposed to solve this problem. The studies presented in this paper exhibited that game theory can be used to learn the convoluted interactions among cognitive users in immensely interfered and shared surroundings.

In [15], a joint power and rate control algorithm for cognitive users in cognitive radio network (CRN) was proposed by using a non-cooperative model based on game theoretical approach. Introducing a pricing factor can also help in maintaining a reasonable data transmission in CR networks. To reduce the interference in a cognitive radio network, power control plays an important role, a sizable work has been carried out in [16, 17]. In [18], power control problems, spectrum sharing and interference adjustment is jointly studied for multiple input multiple output (MIMO) in wireless CRNs. The power under investigation is of non-convex nature.

In [19] the power control problem and beamforming was jointly studied keeping the main focus on reducing the transmitted power of the CR network in such manners that the PU does not suffer from received interference while the QoS requirements for the secondary users are satisfied [19]. While the algorithm ensures the QoS of the communication, however, the power levels may need to adjust frequently in presence of dynamic environment. In this paper, we present a solution to find suitable power levels for CRNs where interference levels changes frequently due to unpredictable channel conditions. The proposed solution models the CRNs with a game, where CRs acts as players of the game. The existence of unique Nash Equilibrium (NE) in the proposed game guarantees that the adaptive transmission power strategy converges to feasible power levels that are required for communication between the source and the destination.

Rest of the paper is organized as follows. In Sect. 2, we describe system model used for evaluation of the proposed solution. In Sect. 3, we discussed the proposed game in detail. In Sect. 4, we evaluate the performance of proposed solution for various network parameters. The conclusions and future directions are discussed in Sect. 5.

\section{System Model}

The system used to evaluate the performance of the proposed solution is shown in Fig. 1. We considered a network scenario where the up-link of cognitive radio network is shared by the cognitive users. As the radio spectrum is only licensed for PU therefore the sharing is only possible if the communication of the primary user is not affected by the interference caused by the CUs during their communication. In the primary network, primary user transmits its data towards dedicated base station over the purchased band with a transmission power $P_{p r i}$. The cognitive network consists of a cognitive base station (CBS) with multiple CUs each transmitting with transmission power $P_{s e c}$ over the same frequency band that is purchased by primary user. We considered all that the base stations and the users are equipped with single antenna. In this case, the SINR of $i$-th CU can be calculated as [20],

$$
\gamma_{i}=\frac{p_{i} h_{i}}{\sum_{j=1, j \neq i}^{N} p_{j} h_{j}+p_{0} h_{0}+\sigma^{2}}
$$

where $h_{i}$ represents the path gain from the cognitive base station (CBS) to the $i$-th cognitive user, $h_{0}$ is the path gain from primary user to cognitive base station. PU and CUs are also linked via channel gain channel $g_{j}$, where $j \in[1, N]$ as shown in Fig. 1 . The variables $p_{0}$ 


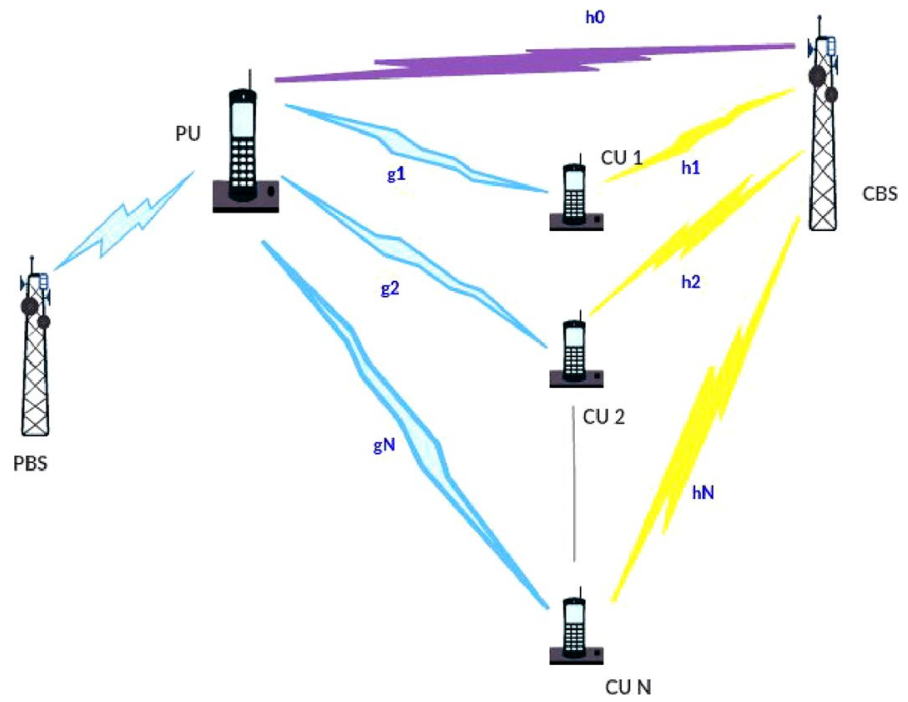

Fig. 1 System model

and $p_{i}$ represents the transmission powers of PU and $i$-th CU, respectively. Moreover, the background noise is additive white Gaussian noise with zero mean and variance $\sigma^{2}$.

In order to ensure that the communication of PU is not affected by the transmission of the cognitive users, the maximum interference from cognitive users must be less than a predefined threshold value $I_{t h}$ defined by the PU. The total received power should satisfy the following constraint [19].

$$
\sum_{i=1}^{N} p_{i} h_{i} \leq I_{t h}
$$

In order to fulfill the quality of service demands of cognitive users, the received signal to interference plus noise ratio (SINR) of each CU should satisfy the following inequality.

$$
\gamma_{i} \geq \gamma_{i}^{\text {th }}
$$

where $\gamma_{i}^{\text {th }}$ is the target SINR required to maintain QoS at the receiver.

\section{Power Control Game}

In this section, we present the game theoretic approach to solve the issues faced by the cognitive radio networks. We will start our discussion with the game model, propose the utility function for proposed network, and proceed with the iterative power control algorithm for optimal power selection in the subsequent sections. Finally, we will prove the existence and uniqueness of Nash Equilibrium (NE) for the proposed power control game. 


\subsection{Game Model}

A game is defined as $G=\{N, A, u()$.$\} where N$ represents the players of the game, $A$ represents the set of strategy space of the players, and $u($.$) represents the utility function$ of each player. In the proposed game model, the components of the game are defined as follows.

Definition 1 (Players $\{N\}$ ) In the proposed game, the CUs are defined as the players of the game.

Definition 2 (Strategy Space $\{A\}$ ) In the proposed game, the strategy space depends on the actions taken by the players while playing the game. If $p_{i} \in s_{i}$ represents the action of $i$-th player, then we can define the strategic space for $N$ players as,

$$
S=p_{1} \times p_{2} \times p_{3} \times \ldots \times p_{N} .
$$

Definition 3 (Payoff Function $\left\{u_{i}\right\}$ ) Payoff function or utility function describes the outcome of players against their actions. The utility of each player depends on its own strategy as well as the strategy of other players in the network.

\subsection{Proposed Utility Function}

In the proposed game, the utility of each node depends on its own strategy as well as the strategy of its opponent, therefore, we can represent the utility function $u($.) of $i$-th CU as,

$$
u_{i}\left(p_{i}, p_{-i}\right)=A_{i} \log \left(\gamma_{i}-\gamma_{i}^{t h}\right)+B_{i} \sqrt{p_{i}^{\max }-p_{i}}
$$

where

$$
p_{i} \in\left[0, p_{i}^{\max }\right]
$$

In Eq. (5), $\gamma_{i}^{\text {th }}$ represents the threshold SINR required for successful communication, $\gamma_{i}$ represents the SINR at node $i, p_{i}^{\max }$ is the maximum transmission power of node $i$, and $p_{i}$ is the current transmission power of node $i$. The variables $A_{i}$ and $B_{i}$ are the weighting factors which are constant and non negative. The value of $A_{i}$ depends on the distance between the $\mathrm{CU}$ and PU that is updated by following equation.

$$
A_{i}^{t}=A_{i}^{t-1} \frac{1}{d^{2}}
$$

where $d$ is the distance between PU and CU. Similarly $B_{i}$ is used to capture the affect of interference experienced at $i$-th CU from other CUs and PU. $B_{i}$ is updated in each iteration as,

$$
B_{i}^{t}=B_{i}^{t-1}\left(\gamma_{i} / \gamma_{i}^{t h}\right)
$$

Equations (5) and (7) shows that the players will increase its transmission power until threshold SINR is reached in order to ensure successful transmission of data towards the destination. 


\subsection{Adaptive Power Control Algorithm}

In this section, we present an adaptive power control algorithm for the proposed game model. The proposed algorithm ensures that the transmission powers of PU and CUs reaches at optimal levels and the game converges to Nash Equilibrium point. A Nash equilibrium (NE) is defined as a point in the game where each participant gets the best payoff in competitive scenario and not a single player can raise its payoff separately by deviating from its own strategy. The NE of the proposed game is defined as follows.

Definition 4 (Nash Equilibrium) A power vector $\mathbf{S}=p_{1}, p_{2}, \ldots, p_{N}$ is Nash equilibrium of the game $\mathbf{G} \forall i \in \mathbf{N}$, if and only if

$$
u_{i}\left(p_{i}^{*}, \mathbf{p}_{-\mathbf{i}}\right) \geq u_{i}\left(p_{i}, \mathbf{p}_{-i}\right), \forall p_{i} \in s_{i}
$$

The power vector $\mathbf{p}_{-\mathbf{i}}$ represents the power levels of all the cognitive users excluding the cognitive user $i, p_{i}$ represents the current power of user $i, p_{i}^{*}$ represents the power level of $i$-th cognitive user at NE point.

\subsubsection{Evaluation of NE Strategies}

In order to calculate the strategies at $\mathrm{NE}$ we take the first order derivation of utility function $u_{i}\left(p_{i}, \mathbf{p}_{-i}\right)$ with respect to $p_{i}$.

$$
\frac{\partial u_{i}}{\partial p_{i}}=\frac{A_{i}}{\gamma_{i}-\gamma_{i}^{t h}} \times \frac{h_{i}}{I_{i}\left(\mathbf{p}_{-i}\right)}-B_{i} \frac{1}{2 \sqrt{p_{i}^{\text {max }}-p_{i}}}
$$

where

$$
I_{i}\left(\mathbf{p}_{-i}\right)=\sum_{j \neq i}^{N} p_{j} h_{j}+p_{0} h_{0}+\sigma^{2}
$$

Equation (10) represents the interference caused by all cognitive users $p_{j} h_{j}$ except the $i$-th $\mathrm{CU}$, the noise caused by primary user $p_{0} h_{0}$ and the background additive white Gaussian noise $\sigma^{2}$. We obtain the following equation by setting the first derivative equal to zero.

$$
\gamma_{i}=\gamma_{i}^{t h}+\frac{2 A_{i} h_{i}}{B_{i} I_{i\left(p_{-i}\right)}} \sqrt{p_{i}^{\text {max }}-p_{i}}
$$

Solving for $p_{i}$ gives optimal power level for $i$-th CU as,

$$
p_{i}=\frac{I_{i}\left(p_{-i}\right)}{h_{i}} \gamma_{i}^{t h}+\frac{2 A_{i}}{B_{i}} \sqrt{p_{i}^{\max }-p_{i}}
$$

By putting the value of interference for the channel model defined in Sect. 2, we get,

$$
p_{i}^{m+1}=\frac{p_{i}^{(m)}}{\gamma_{i}^{(m)}} \gamma_{i}^{t h}+\frac{2 A_{i}}{B_{i}} \sqrt{p_{i}^{\max }-p_{i}^{(m)}}
$$


The power iteration function for $i$-th user can be written as,

$$
p_{i}^{m+1}= \begin{cases}\frac{p_{i}^{(m)}}{\gamma_{i}^{(m)}} \gamma_{i}^{t h}+\frac{2 A_{i}}{B_{i}} \sqrt{p_{i}^{\max }-p_{i}^{(m)}} & 0<p_{i}^{(m+1)}<p_{i}^{\max } \\ p_{i}^{\max } & p_{i}^{(m+1)} \geq p_{i}^{\max }\end{cases}
$$

Each CU adjusts its power level using Eq. (14) in each iteration until the algorithm converges to optimal power levels. The step-by-step procedure of the proposed scheme is described in Algorithm 1.

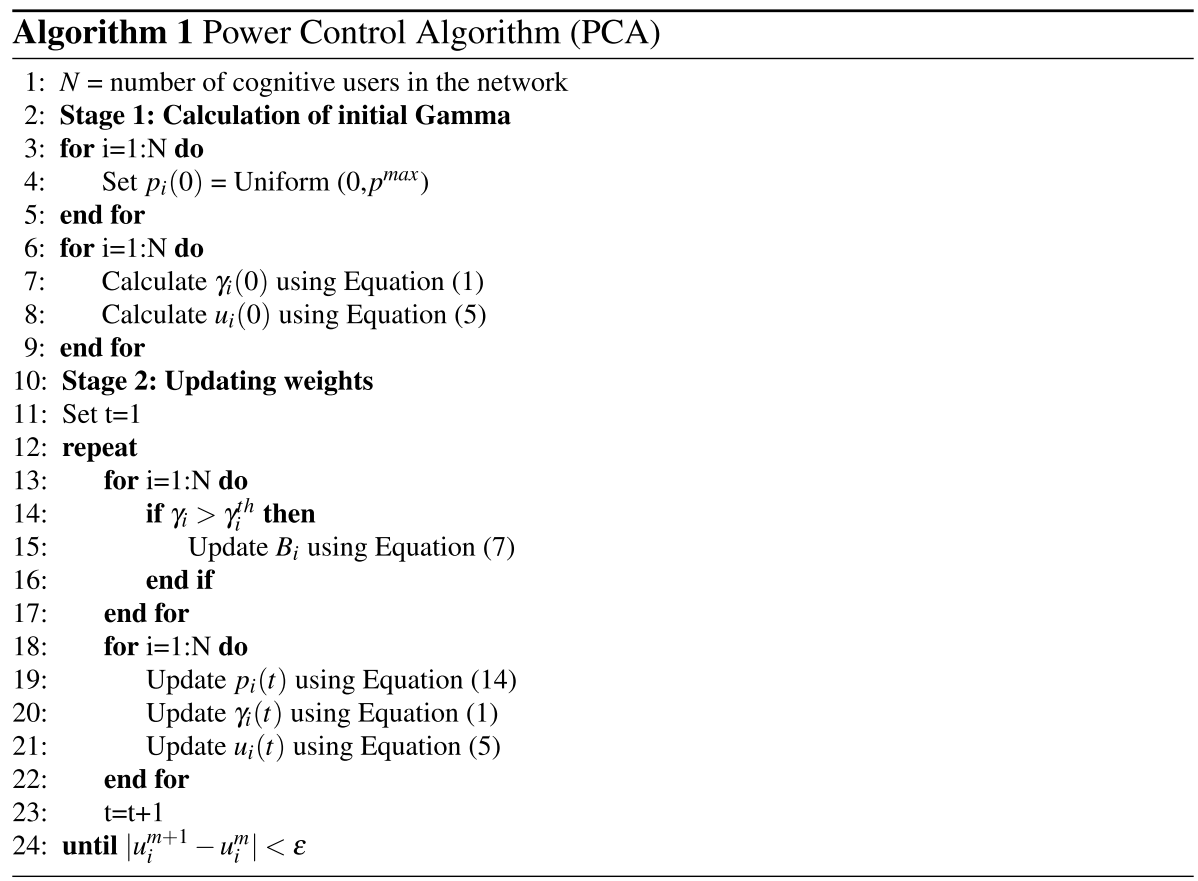

\subsection{NE of Proposed Game}

In this section, we discuss the existence and uniqueness of NE obtained by applying the Power Control Algorithm (PCA) proposed in previous section.

Theorem 1 (Existence of NE) The proposed utility function ensures at least one NE point in the game.

Proof A power control game guarantees existence of at least one NE point if following conditions are satisfied [17].

1. The transmission power vector $p_{i}$ must be a non-empty set.

2. The utility function should be quasi-concave. 
3. The utility function should be continuous.

As the users selects their initial transmission power uniformly from a non-empty set of power levels and update powers based on the SINR value at previous time instant, therefore, the resultant power vector will be non-empty. The utility function proposed in Eq. (5) is a combination of well-know continuous functions, as a result, the proposed utility function is continuous. Now we will prove the quasi-concaveness of the proposed utility function in order to complete the proof.

To prove that the proposed utility function is quasi concave, we take the double derivative of utility $u_{i}\left(p_{i}, p_{-i}\right)$ with respect to $p_{i}$ which gives,

$$
\frac{\partial^{2} u_{i}\left(p_{i}, p_{-i}\right)}{\partial^{2} p_{i}}=\frac{A_{i}}{\left(\gamma_{i}-\gamma_{i}^{\text {th }}\right)^{2}} \times \frac{-h_{i}^{2}}{I_{i}^{2}\left(p_{-i}\right)}-B_{i} \frac{1}{4\left(\sqrt{p_{i}^{\max }-p_{i}}\right)^{3}}
$$

While Algorithm 1 ensures that $p_{i}<p_{i}^{\max }$ and $\gamma_{i}>\gamma_{i}^{\text {th }}$, therefore, $\left.\frac{\partial^{2} u_{i}\left(p_{i}, \mathbf{p}_{-i}\right)}{\partial^{2} p_{i}}<0\right)$, which means that the utility $u_{i}\left(p_{i}, \mathbf{p}_{-i}\right)$ is concave in nature with respect to $p_{i}$. This proves the theorem.

Theorem 2 The NE point in the proposed game is unique.

Proof In order to hold a unique NE point, the reaction function of the players should satisfy the following conditions. [21].

1. Positivity: $f\left(p_{i}\right)>0 \forall i \in N$.

2. Monoticity: if $p_{i}>p_{i}^{\prime}$ then $f\left(p_{i}\right)>f\left(p_{i}^{\prime}\right)$.

The reaction function $f$ of the player $i$ is the optimal power power level selected against the best power levels of other player. The reaction function of $i$-th player depends on its own transmission power as well as the transmission power of its opponents that can be written as,

$$
f\left(p_{i}\right)=\frac{2 A_{i}}{B_{i}} \sqrt{p_{i}^{\max }-p_{i}}+\frac{p_{i} \gamma_{i}}{\gamma_{i}^{t h}}
$$

While $\gamma_{i}, \gamma_{i}^{\text {th }}, A_{i}, B_{i}, p_{i}$, and $p_{i}^{\max }$ are positive real numbers, also, Algorithm 1 ensures that $p_{i}<p_{i}^{\max }$, therefore, the proposed reaction function is positive. For a monotonic function, $f\left(p_{i}\right)>f\left(p_{i}^{\prime}\right)$ if $p_{i}>p_{i}^{\prime}$. Suppose that the user $i$ selects the power $p_{i}^{\prime}>p_{i}$ instead of $p_{i}$, then its reaction function can be written as,

$$
f\left(p_{i}^{\prime}\right)=\frac{2 A_{i}}{B_{i}} \sqrt{p_{i}^{\max }-p_{i}^{\prime}}+\frac{p_{i}^{\prime} \gamma_{i}}{\gamma_{i}^{t h}}
$$

As $p_{i}^{\prime}>p_{i}$, therefore, the term $\frac{p_{i}^{\prime} \gamma_{i}}{\gamma_{i}^{t h}}>\frac{p_{i} \gamma_{i}}{\gamma_{i}^{t h}}$, and $f\left(p_{i}^{\prime}\right)>f\left(p_{i}\right)$. This completes the proof. 


\section{Simulation Results}

In this section, we present the results of simulations to evaluate the performance of proposed scheme. We present a comparative analysis of the proposed algorithm with previously proposed power control algorithms designed for cognitive networks for various network parameters.

\subsection{Simulation Setup}

In the simulation setup, we consider one PU and 8 cognitive users simultaneously transmitting over same frequency band. The cognitive users can communicate through a cognitive base station known as CBS in addition to direct communication with each other. We consider static network for simulation purposed however the results can easily be extended for random node position. The channel between the CUs and CBS $h(i)$ follows the decaying path-loss law which is given as,

$$
h_{i}=\frac{1}{d_{i}^{4}}
$$

where $d_{i}$ denotes the distance between $i$-th cognitive user and CBS. For the static network of eight users, following distance matrix is used.

$$
d=[550,580,600,620,640,950,1000,1050]
$$

These distances are taken in order to analyze the SINR of CUs in different vicinity of CBS. The channel gain between the cognitive users and the primary user is $g(i)$ and is supposed as a Rayleigh flat fading channel. As there is no line of sight between the transmitter and the receiver so Rayleigh fading is very suitable in such scenarios. To initiate the communication, the initial transmission powers are selected for all cognitive users has been chosen from the set of available transmission powers. The upper limit for the transmit power of cognitive users is set to $p_{i}^{\max }=0.5 \mathrm{~W}$. We set the initial power randomly from interval $[0,0.5 \mathrm{~W}]$. We selected the transmission power of PU as $P_{0}=0.005 \mathrm{~W}$ and the SINR threshold $\gamma_{i}^{\text {th }}$ is selected as $7 d B$. For simplicity, we are considering the background noise as AWGN (additive white Gaussian noise) and value of $\sigma^{2}$ is $\sigma^{2}=5 \times 10^{(-15)}$. The rest of parameters are $\epsilon=10^{-15}$ and $\frac{2 A_{i}}{B_{i}}=3 \times 10^{-4}$.

\subsection{Results}

In this section, we discuss the results for various network scenarios. The power levels achieved by each user under proposed scheme are shown in Fig. 2. It is clear from the graph that the transmission power of each user depends on its location form CBS. The users that lies in high interference region increases their transmission power in order to achieve threshold SINR value while the users that are near to CBS transmits on low transmission power in order to save their energy.

In Fig. 3, we plot the SINR at the nodes when transmitting at optimal power levels. The graph demonstrates the threshold SINR is achieved within 10-15 iterations. The convergence rate increases with increases in number of users in the network. 
Fig. 2 Power graphs of proposed algorithm

Fig. 3 SINR results of proposed algorithm comparison with Koskie and Gajic's algorithm also known as K-G algorithm [22] and ANCPCA [23] in Figs. 4 and 5. The results show that the users that are placed closer to the CBS have better SINR then the three user which are placed far from CBS.

The comparison of K-G and ANCPA in Figs. 4 and 5 shows that, in KG, all the CUs are not fulfilling the QoS requirement while in ANCPCA all CUs are fulfilling the QoS requirement however the convergence of the algorithmis slow.

The comparison between Figs. 3, 4 and 5 shows that the convergence of the proposed algorithm is fast as compared to K-G and ANCPA algorithm while meeting the QoS demand. 
Fig. 4 SINR results of ANCPCA

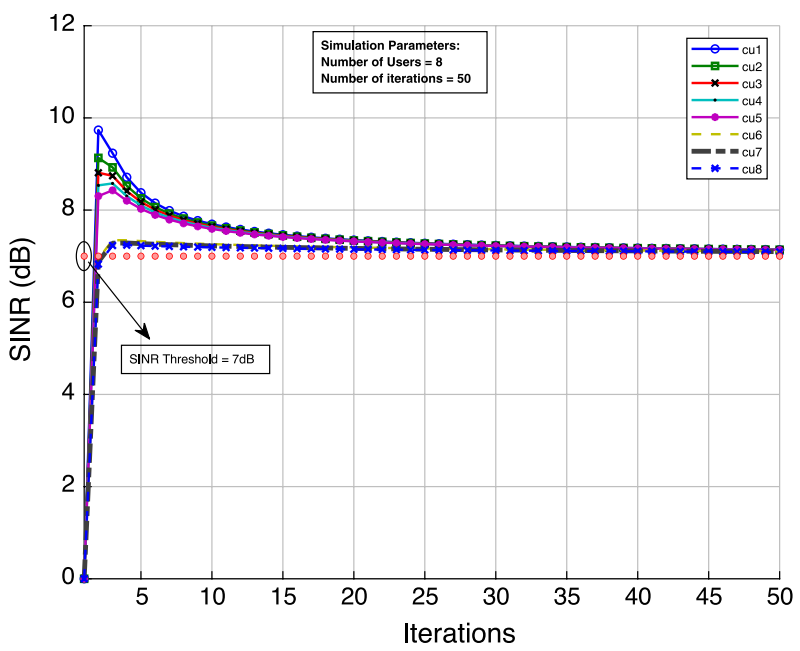

Fig. 5 K-G algorithm

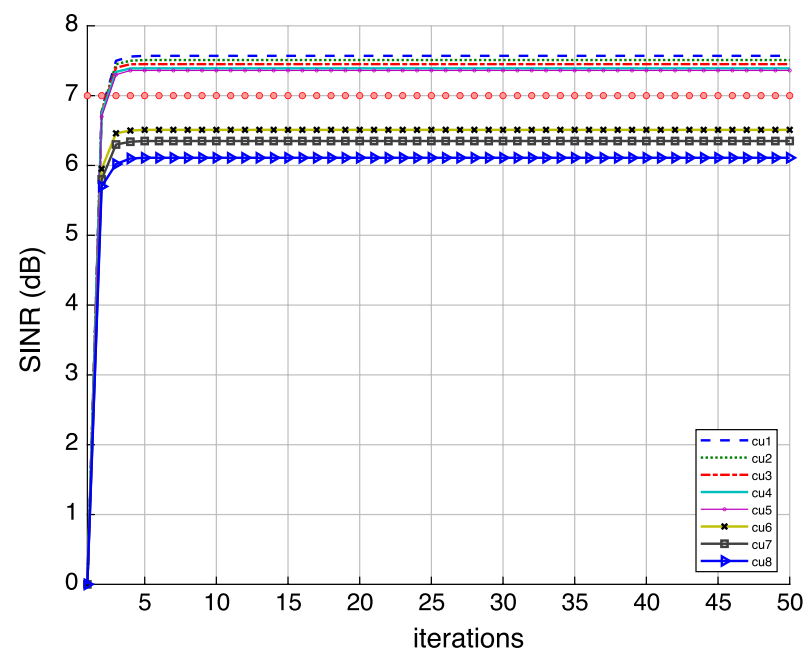

\section{Conclusion and Future Work}

In this paper, we show that the CR networks have limited resources and these resources need to be utilized properly to make the network more practical.

The first is to reduce the power expenditure and protect the primary user from the harmful interference. QoS requirements of the cognitive network should also be met. We modeled a non-cooperative power control game to solve the power allocation problem [24]. An adaptive, iterative power control algorithm is proposed to help the CUs to chose an optimal power level to make the communication possible.

The outcome of a game is analyzed by achieving a NE. The existence of NE plus it uniqueness is also proved mathematically. Last portion of this work includes the simulation results and it comparison with previous work that has been done in the same domain. The presented graphs show that the SINR is improved by keeping check on power consumption. Its comparison with the different algorithms shows the improvement in SINR for each 
$\mathrm{CU}$. The proposed algorithm efficiently uses the power and solves the problem of near-far effect.

In future we can extend this work to networks consisting of multiple primary users and multiple cognitive users. Another extension of this work could be application of this algorithm for mobile users. Similar work can be done for multiple antennas.

Funding Open access funding provided by Università degli Studi di Catania within the CRUI-CARE Agreement.

Open Access This article is licensed under a Creative Commons Attribution 4.0 International License, which permits use, sharing, adaptation, distribution and reproduction in any medium or format, as long as you give appropriate credit to the original author(s) and the source, provide a link to the Creative Commons licence, and indicate if changes were made. The images or other third party material in this article are included in the article's Creative Commons licence, unless indicated otherwise in a credit line to the material. If material is not included in the article's Creative Commons licence and your intended use is not permitted by statutory regulation or exceeds the permitted use, you will need to obtain permission directly from the copyright holder. To view a copy of this licence, visit http://creativecommons.org/licenses/by/4.0/.

\section{References}

1. Wang, B., Wu, Y., \& Liu, K. R. (2010). Game theory for cognitive radio networks: An overview. Computer Networks, 54(14), 2537.

2. Loscri, V., Maskooki, A., Mitton, N., \& Vegni, A. M. (2015). Wireless cognitive network technologies and protocols. In Modeling and Simulation of Computer Networks and Systems (pp. 119-153). Amsterdam: Elsevier.

3. Valenta, V., Fedra, Z., Marsalek, R., Baudoin, G., Villegas, M. (2009). Towards cognitive radio networks: Spectrum utilization measurements in suburb environment. In 2009 IEEE Radio and Wireless Symposium (IEEE) (pp. 352-355).

4. Wang, J., Ghosh, M., \& Challapali, K. (2011). Emerging cognitive radio applications: A survey. IEEE Communications Magazine, 49, 3.

5. Shukla, A. (2007). Cognitive Radio Technology: A Study for of com-Volume 1. GNU Radio Companion-usage Tips.

6. Haykin, S., et al. (2005). Cognitive radio: brain-empowered wireless communications. IEEE Journal on Selected Areas in Communications, 23(2), 201.

7. Ghasemi, A., \& Sousa, E. S. (2007). Fundamental limits of spectrum-sharing in fading environments. IEEE Transactions on Wireless Communications, 6, 2.

8. Hoang, A. T., \& Liang, Y. C. (2008). Downlink channel assignment and power control for cognitive radio networks. IEEE Transactions on Wireless Communications, 7, 8.

9. Zhang, R., Liang, Y. C., \& Cui, S. (2010). Dynamic resource allocation in cognitive radio networks. IEEE Signal Processing Magazine, 27(3), 102.

10. Kang, X., Liang, Y. C., Nallanathan, A., Garg, H. K., \& Zhang, R. (2009). Optimal power allocation for fading channels in cognitive radio networks: Ergodic capacity and outage capacity. IEEE Transactions on Wireless Communications, 8(2), 940.

11. Zhao, N., \& Sun, H. (2011). Robust power control for cognitive radio in spectrum underlay networks. KSII Transactions on Internet and Information Systems, $5,7$.

12. Bedeer, E., Dobre, O. A., Ahmed, M. H., \& Baddour, K. E. (2014). A multiobjective optimization approach for optimal link adaptation of OFDM-based cognitive radio systems with imperfect spectrum sensing. IEEE Transactions on Wireless Communications, 13(4), 2339.

13. Li, D., Dai, X., Zhang, H., (2008). Game theoretic analysis of joint rate and power control in cognitive radio networks. In Communications, Circuits and Systems, 2008. ICCCAS 2008. International Conference on (IEEE) (pp. 319-322).

14. Abdul-Ghafoor, O. B., Ismail, M., Nordin, R., \& El-Saleh, A. A. (2013). Resource allocation in spectrum sharing ad-hoc cognitive radio networks based on game theory: An overview. TIIS, 7(12), 2957. 
15. Zhou, P., Yuan, W., Liu, W., Cheng, W. (2008). Joint power and rate control in cognitive radio networks: A game-theoretical approach. In Communications, 2008. ICC'08. IEEE International Conference on (IEEE) (pp. 3296-3301).

16. Sun, A.W., Zhang, H. (2012). An improved power control algorithm in cognitive radio system. In Communication Technology (ICCT), 2012 IEEE 14th International Conference on (IEEE) (pp. 1193-1197).

17. Li, F., Tan, X., \& Wang, L. (2011). A new game algorithm for power control in cognitive radio networks. IEEE Transactions on Vehicular Technology, 60(9), 4384.

18. Nguyen, D.N., Krunz, M. (2012). Spectrum management and power allocation in MIMO cognitive networks. In INFOCOM, 2012 Proceedings IEEE (IEEE) (pp. 2023-2031).

19. Islam, H., \& Liang, Y.C., Hoang, A.T. (2008). Downlink channel assignment and power control for cognitive radio networks. IEEE transactions on wireless communications, 7(7).

20. Waqas, A., Mahmood, H. (2013). Interference aware adaptive routing for cdma based mobile ad hoc networks. In 2013 4th international conference on intelligent systems, modelling and simulation (IEEE) (pp. 492-496).

21. Zhao, F., Li, B., Chen, H., \& Lv, X. (2013). Joint beamforming and power allocation for cognitive MIMO systems under imperfect CSI based on game theory. Wireless Personal Communications, 73(3), 679.

22. Koskie, S., \& Gajic, Z. (2005). A Nash game algorithm for SIR-based power control in $3 \mathrm{G}$ wireless CDMA networks. IEEE/ACM Transactions on Networking, 13(5), 1017.

23. Yang, G., Li, B., Tan, X., \& Wang, X. (2015). Adaptive power control algorithm in cognitive radio based on game theory. IET Communications, 9(15), 1807.

24. Nash, J. (1951). Non-cooperative games. Annals of Mathematics, 54(2), 286-295.

Publisher's Note Springer Nature remains neutral with regard to jurisdictional claims in published maps and institutional affiliations.
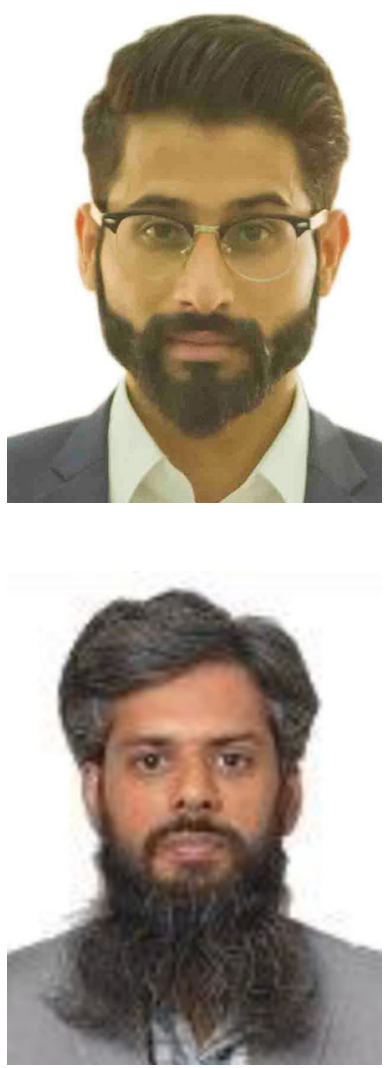

Waqas Gulzar received his Master of Philosophy (M.Phil) degree in Electronics from Quaid-i-Azam University Islamabad, Pakistan. Currently, doing his Ph.D. from the Department of Electrical Electronic and Computer Engineering, Università degli Studi di Catania, Italy. His research interests include Communication Systems, Game Theory, and Artificial Intelligence.

Abdullah Waqas received the Ph.D. degree in Electronics from Quaidi-Azam University Islamabad, Pakistan. His research interests include communication systems, Ad hoc networks Game Theory Distributed Networks Machine Psychology. He is currently working as an Assistant Professor at Department of Electrical Engineering, National University of Technology, Sector I-12, Islamabad, Pakistan. 

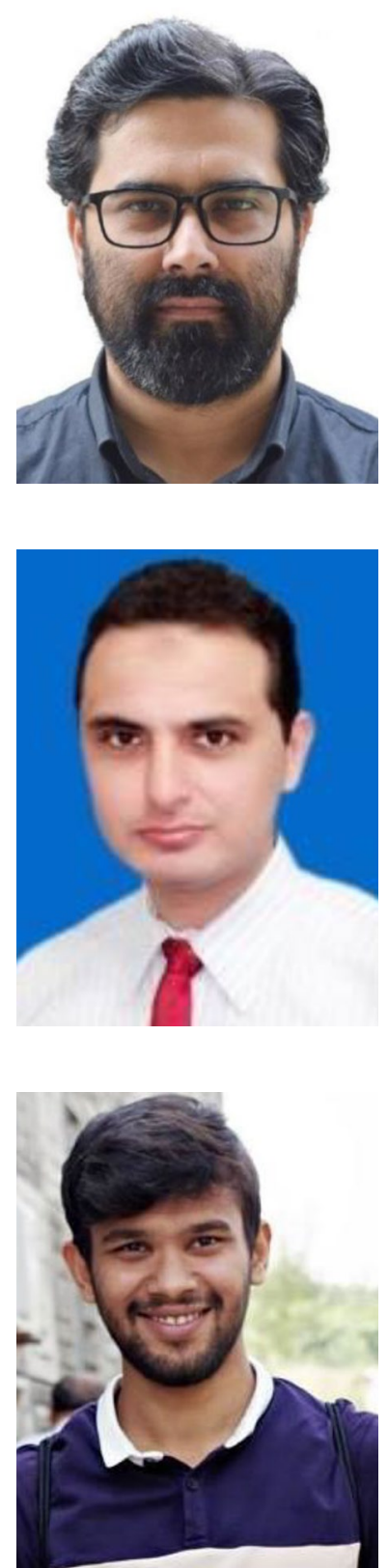

Hammad Dilpazir received his Ph.D. degree in Electronics from Quaid-i-Azam University Islamabad, Pakistan. His areas of interest include Signal processing, Information Theory, Data fusion, Artificial Intelligence, and Internet of Things. He is currently working as an Assistant Professor at Department of Electrical Engineering, National University of Modern Languages, Islamabad Pakistan.

Anwar Khan received his Ph.D. degree in Electronics from Quaid-iAzam University Islamabad, Pakistan. His areas of interest include Signal processing, Information Theory, Game Theory, and Wireless Communication.

Ashfaq Alam received his Master of Philosophy (M.Phil) degree in Electronics from Quaid-i-Azam University Islamabad, Pakistan. His areas of interest include Signal processing, Information Theory, Image Processing, and Wireless Communication. 


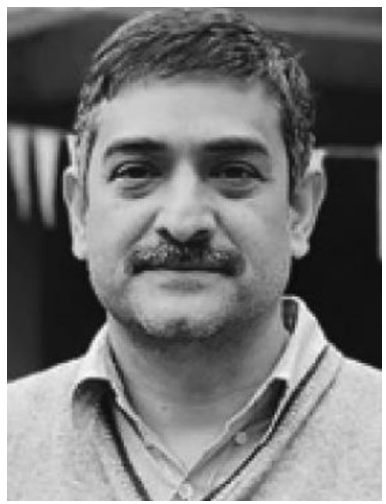

Hasan Mahmood received the Ph.D. degree in electrical engineering in USA, in 2007. His research interests include communication systems, Ad hoc networks, channel coding, and computer architecture. 